\title{
The Discussion About the Application of Modern Automotive Electronics Technology
}

\author{
Yong-jie Zhang \\ Yunnan Jiaotong College, Kunming 650500, P. R. China .
}

Keywords: Automobile Electronic Technology, Application, Trend in Development.

\begin{abstract}
Electronic and information technology's progress and promote inevitably cause related industries' technology innovation. To master and develop of advanced technology and methods is a powerful guarantee that enterprises can seize the initiative in an increasingly competitive global market. As one of the fastest growing and the most rapidly evolving industries, automobile industry must keep enhancing the level of production management to create products that meet the market demand with people life style and consumption idea transformation, the choice of travel transport diversified, and more and more requirements demanded. Nowadays the perfect combination of the electronic technology and automobile manufacturing is of great significance for automobile enterprises. Based on the problems related to the research of automobile electronic technology, its development trend were discussed in this paper.
\end{abstract}

\section{Introduction}

The perfect combination of electronic information technology and automobile production is the latest theory of automobile design and manufacture. It can make mechanical systems, operating systems and electronic information technology of vehicles operate harmoniously, and make products smarter, safer and have a better interface. The enterprises should stick to market-oriented strategy, grasp the latest trend of the market, and acquaintance cutting-edge technologies to maintain the competitive advantages. The application of electronic technology has become an important indicator to measure the level of automobile manufacturing. So the research on this field is of great practical significance.

\section{Overview of Modern Automotive Electronic Technology}

The rapid development of electronic technology has given rise to the birth of the automobile electronic technology. Form transistor, integrated circuit, large scale integrated circuit to super scale integrated circuit, A variety of electronic products appear in people's daily lives because of the progress in technology, such as computers. The penetration of information technology makes the automobile electronic technology more meaningful, and has great significance for the automobile function and the whole performance.

\section{The Concept of Automobile Electronic Technology}

In simple terms, automotive electronic technology is to design, manufacture, application combined with electronic technology, to make the automobile overall performance improvement. Automobile electronic technology generally contains two aspects. The first is the car application of electronic information technology. They contain electronic facilities which can operate independently and don't have a significant impact on the operation of the vehicle. Their main functions are information processing, navigation, communication and entertainment functions by using on-board computer. The second is that the cars' own functional electronic control technology. They are very important to the start-up, operation, control, etc. And they contain sensors, ECU and actuators which assist the mechanical device to complete the automatic loop control. 


\section{The Characteristics of Modern Automobile Electronic Technology}

According to the information already mastered, the international advanced automotive electronic technology has the following characteristics:

Improvement of Driving Safety. The basic idea of automobile design is to be safe and reliable. The use of modern electronic technology in the control performance of the car, can greatly enhance the safety of driving and riding a car. Active control system and passive control system are used in electronic technology. Active control system can reduce the driver's fatigue, and make driving becomes a more relaxed and convenient experience. Due to the intelligent and sensitive electronic system, once the driving accident happening, it can take appropriate emergency measures in a timely manner, to the maximum extent to avoid the damage to the drivers and passengers. Otherwise, traction system and ABS system can effectively reduce the collision accident, the dangerous inhibition in the initial stage.

Perfection of Control Function. The innovation of automotive technology is largely reflected in the improvement of the control system. From the ABS system to ASR system, traction control system, braking force distribution system, ESP system and so on, they all reflects the gradual improvement of control system and improve the ease of operation and safety of driving.

The driver detection system is an advanced system based on information collection reflection theory, which can monitor the status and behavior of the driver. When abnormal situation happened, the system timely warned the driver. Early warning system strengthen the man-machine interface, and use a clearer display and voice prompt. Tire pressure detection system can be real-time detection of tire pressure and temperature, can effectively prevent the burst even more serious collision accidents.

Enhancement of Low Carbon Concept. Low carbon concept is a new concept of development in the manufacturing industry, construction industry and so on, adapted to the development needs of modern society. With the rapid development of automobile industry, a series of serious social problems and ecological problems are inevitable, and the destruction of energy and environment has increasingly become the focus of attention. The use of electronic technology can effectively improve the efficiency of the engine work, both achieve the reduction of the fuel consumption, as it would be helpful to solve the energy crisis. At the same time due to the efficient use of fuel, it can reduce the pollution of the gas and toxic emissions output and the pollution to the environment.

\section{Development History of Automobile Electronic Technology}

Since the combination of electronic technology and automobile industry, the development of the automobile electronic technology has generally experienced four stages of development. The first stage is from 1950s to early 70 s. This period of the main development object is composed of discrete components and integrated circuit of automotive electronic products, will be the most conventional cars machinery replacement, completed the first combination of cars and electronics. The second stage is from mid $70 \mathrm{~s}$ to mid $80 \mathrm{~s}$. The main content of this stage is the development of the independent system of automobile. Some electronic devices such as electronic control fuel injection system and ABS system started application in automotive systems, fundamentally solved what mechanical system could not solve. The third stage is from the middle of 80 s to the middle of 90 s. During this period, more powerful electronic devices was invented, such as engine control and automatic transmission control and etc. The main results of the research focus on the integrated system and the crisis control field of the overall performance of the vehicle. The fourth stage begins in the middle of 90s. Information technology has been introduced into the automotive design since 1990s. This realized the intelligent and networked control of the vehicle, and has been greatly improved in the aspects of safety performance, resource sharing and so on. 


\section{Application of Modern Automobile Electronic Technology}

According to affect the performance of automobile electronic technology can be divided into two categories, namely the automotive electronic control system and the on-board electronic systems. The vehicle electronic device has little effect on the overall performance of the vehicle, which is mainly to provide users with a better multi-functional auxiliary facilities and a user-friendly device. Automotive electronic control device is the guarantee of the performance of the automotive system. At present, the mature electronic control system include engine electronic control, automobile body electronic control, chassis electronic control and information transmission, etc.

\section{Engine Electronic Control}

Electronic Controlled Fuel Injection Device. The traditional fuel injection system, such as mechanical fuel injection device and electromechanical hybrid fuel injection device, has been eliminated in the modern automobile design because of its poor performance and efficiency, and been replaced by the excellent performance of electronically controlled fuel injection device. The development of the fuel injection control system began in 1960s, and now the technology has reached a mature stage. Compared with the traditional fuel injection technology, the electronic control injection can achieve the best air fuel ratio, greatly improve the fuel haze, and the positioning is very accurate, so that the engine performance has been greatly improved.

Electronic Ignition Device. From the contact type, the ordinary non-contact type to the integrated circuit type and the microcomputer control electronic ignition device, the application of the ignition system has a history of over a century. The performance of the devices are getting better and better, so that the ignition time of the engine is controlled in an excellent range, which greatly develops the potential of the engine.

Other Engine Electronic Devices. In addition to these electronic technology and engine performance are closely linked, have different levels of electronic technology being used in automobile manufacturing, such as idle speed control system, electric pumps, solar term valve timing, engine turbo system and system self-diagnosis and etc.

\section{Body Electronic Control}

Air Conditioning Control System. With the requirements of the modern society for human habitation and travel comfort, the automobile air conditioning has become an indispensable facility, and the performance of the air conditioner is directly related to the customer's attraction and loyalty. The main principle of the automatic air conditioning system is to use the information recorded by the temperature sensor inside and outside the body to calculate the wind temperature to achieve the ideal.

Information Display System. At present, the information display system is being further improved. This system consists of three parts: the vehicle condition detecting device, vehicle computer, electronic instrument. The three parts worked orderly. Through the detection of liquid level, temperature, pressure, light, condition detection device transmits the data to the on-board computer analysis, troubleshooting, presented to the driver to form a good interface.

Airbag Control System. The airbag control system belongs to the passive protection device, which is a powerful barrier for the driver to protect the safety of the vehicle after the accident. It is composed of three parts: trigger device, gas generator and air bag. Among them, the technical requirements of the airbag triggering device are relatively high, which requires a high degree of flexibility and intelligence. The whole system needs a strong coordination control ability to ensure that the airbag is in the best working condition at all times.

\section{Chassis Electronic Control}

Anti-lock Braking System. ABS is the earliest development of chassis control system, but also the application of electronic technology safety device most widely. It is mainly through the control of 
the wheel lock braking to ensure the wheel and the ground can reach the optimal sliding rate. When the slip ratio is controlled between $15 \%$ and $20 \%$, the wheel and the ground can achieve the vertical peak adhesion coefficient and the large lateral adhesion coefficient when the vehicle is braked under various road conditions. So as to ensure that the vehicle does not completely slip insecurity.

Electronic Transmission Devices. The biggest advantage of the electronic transmission is that the acceleration performance is strong, the sensitivity is high, at the same time, it can carry on the automatic control according to the road condition and the traveling load, which has guaranteed the driving power and the reliability. According to the parameters of the engine and the driver, the system can automatically adjust the gear lever, so as to realize the optimal control of the shift.

Electronic Steering Assist System. This system adopts DC motor, battery and motor provides the power to replace the traditional hydraulic cylinder booster system, the weight is lighter, smaller and more powerful, greatly enhance the ability of automobile steering, increase the steering characteristics and corresponding maneuverability at low speeds the car.

\section{Information Transfer}

Information Display and Alarm. Information display system is the collection, analysis and reporting of information on the condition, to provide reference data for real effective drivers, so as to make the appropriate response, to ensure the stability of vehicle driving safety and the whole car. It is the basic application system to prevent driving accidents and vehicle maintenance.

Voice Information System. The voice information system includes two aspects: voice control and warning. When the vehicle is not in normal condition, the electronic control equipment through the logic of reasoning to determine the integration of information, in the form of voice to inform the driver. Voice control is the voice of the driver input system, the realization of the function of the car control.

Navigation system. Navigation system is a kind of electronic system integration of the road information warning, warning for the remote and strange voice traffic driving design, can provide the best route and related information for drivers.

\section{Development Trend of Automotive Electronic Technology}

\section{Sensor Technology}

The electronic technology of automobile sensor is the key technology to realize the high end, automation and intelligence. With require of the improvement of the convenience and automatic control function, the sensor technology must be combined with the automobile technology. At present, the function of the automobile sensors have limits, and they can't make the car go to the design concept of the future, to achieve the degree of man-machine control mutual aid. The development of the sensor should aim at the development of multi-function, high integration and intelligence. It can respond to the automobile and driver information in real time, and it can also maintain the stability and efficiency of the system under special circumstances.

\section{Micro-processing Technology}

The on-board computer is the center of the whole electronic control system, which is responsible for receiving, analyzing and judging the information, and then controlling the other components. Along with the automobile composite facilities and centralized development, computer information processing quantity increasing, the complex degree is deepened ceaselessly, at the same time to ensure the accuracy and stability of computer performance, the microcomputer processing challenges technology. It is of great practical significance to develop the technology of microcomputer control with multiple synchronous control, $\mathrm{A} / \mathrm{D}$ and $\mathrm{D} / \mathrm{A}$, high input / output. 


\section{Vehicle Network Technology}

The development of information technology has been extended to many aspects of life. An automotive technology, the realization of effective communication and resource information transmission and sharing will be a major trend in the development of automotive electronics technology. By reducing the wiring, weight and quantity control of vehicle network facilities, enhancing the effectiveness of the control network, the network information transmission can be more secure, and improve the overall performance of the automobile, the simplified vehicle maintenance work, increasing the driving of human nature

\section{Bus Technology}

Bus technology is in the car each unit of the electronic control module and intelligent sensing equipment and intelligent instrument connected, so as to construct automobile internal LAN. The development of bus technology will bring great convenience for automobile manufacturing and driving. First of all, the number of wires and connection points in the unit and unit internal are reduced greatly, which makes the circuit layout more simple and improves the reliability and maintainability of the system. Secondly, the application of the common sensors, can achieve data sharing of the information and enhance the intelligence and operational efficiency of the system. Finally, the application of bus technology will greatly improve the flexibility of the system.

\section{References}

[1] Long Yu. Application status and development trend of modern automotive electronic technology [J] Mechanical management and development, 2009 (04)

[2] Zhao Tongyu. Characteristics and development trend of modern automotive electronic technology [J]. Value engineering, 2011 (06)

[3] Zhao Yutian. Modern automotive electronic technology and its application in instrument analysis [J]. Electronic testing, 2012 (09)

[4] Fang Yong. Application and development trend of automobile electronic technology [J]. Knowledge economy, 2012 (09)

[5] Zhuang Yanxia. Application and development trend of automobile electronic technology [J]. Agricultural equipment and vehicle engineering, 2009 (02)

[6] Liu Ruixue. In the modern automobile electronic technology application and development of [J]. Economic Research Journal, 2010 (19)

[7] Cai Shu, Mou clear. The development of automotive electronic technology and application of [J]. Public technology, 2012 (07)

[8] Zhu Hua. Development of modern automotive electronic control technology [J]. Modern components, 2011 (05) 